morbidity. In patients with RA it has been stablished a high prevalence of comorbidities and their risk factors (1).

Objectives: The aim of this study was to evaluate the prevalence of comorbidities in Colombian patients with RA enrolled in an educational multidisciplinary program and possible correlation with disease activity

Methods: We performed a cross-sectional study; we included patients with confirmed diagnosis of rheumatoid arthritis in a specialized RA center. We collected sociodemographic data, and markers of disease activity DAS28. We collected data regarding the history of comorbidities such as hypertension, dyslipidemia, osteoporosis, type 2 diabetes mellitus, hypothyroidism, malignancies, among others. We performed a descriptive analysis, variables with a normal distribution were described using mean and standard deviation (SD), and non- normal distributed variables were described using median and interquartile range. Categorical variables were presented as rates. We evaluated the relationship between disease activity and comorbidities.

Results: We included 251 patients; mean age was $59 \pm 9.8$ years old, with a high proportion of women 93\%; median disease duration was 15 years RIQ (8-20); in this study, $145(65 \%)$ of patients were in remission; $35(11 \%)$ had low, $44(20 \%)$ moderate and 10 (4\%) high disease activity. Regarding pharmacological therapy, $55 \%$ were receiving conventional DMARDs. The prevalence of comorbidities was $85 \%$, the most common were high blood pressure $25 \%$ followed by hypothyroidism $12 \%$ and diabetes $10 \%, 0.7 \%$ of patients had malignancies such as thyroid cancer or breast cancer, $1.29 \%$ of patients had renal comorbidities. Among comorbidities related to RA $30 \%$ had osteoporosis and $20 \%$ arthrosis. We did not find a statistical association between DAS28 and comorbidities.

Conclusion: As other studies have shown, there is a high prevalence of comorbidities among RA patients, mainly high blood pressure. Due to the above, it is relevant to evaluate the risks factors of patients with $\mathrm{RA}$, especially cardiovascular risks. We consider that a multidisciplinary program represents an opportunity not only to educate patients about healthy life styles and the management of RA, but also other diseases in order to increase the empowering of the health status in these poly pathological patients(2).

References:

[1] Gullick NJ, Scott DL. Co-morbidities in established rheumatoid arthritis. Best practice \& research Clinical rheumatology. 2011;25(4):469-83.

[2] Galarza-Delgado DA, Azpiri-Lopez JR, Colunga-Pedraza IJ, Cardenas-de la Garza JA, Vera-Pineda R, Wah-Suarez M, et al. Prevalence of comorbidities in Mexican mestizo patients with rheumatoid arthritis. Rheumatology international. 2017;37(9):1507-11.

Acknowledgments: This project has been funded by a collaboration between the Ministry of Science, Technology and Innovation COLCIENCIAS (contract 746-2018), the Fundación Universitaria de Ciencias de la Salud and Biomab Center for Rheumatoid Arthrtitis

Disclosure of Interests: Michael Cabrera: None declared, Fernando Rodriguez: None declared, Diana Buitrago-Garcia: None declared, GUILLERMO SÁNCHEZ: None declared, Pedro Santos-Moreno Grant/research support from: I have received research grants from Abbvie, Biopas-UCB, Janssen, Novartis, Pfizer., Speakers bureau: I have been a speaker for Abbvie, Biopas-UCB, Janssen, Lilly, Novartis, Pfizer, Roche, Sanofi.

DOI: 10.1136/annrheumdis-2020-eular.5024

\section{AB1297-HPR BONE AND MINERAL METABOLISM IN SPONDYLOARTHRITIS}

D. Castro-Corredor ${ }^{1}$, M. A. Ramírez Huaranga ${ }^{2}$, A. I. Rebollo Giménez ${ }^{2}$, M. D. Mínguez Sánchez ${ }^{2}$, J. Anino-Fernández ${ }^{2}$, I. M. De Lara Simón ${ }^{2}$, J. L. Cuadra Díaz ${ }^{2}{ }^{1}$ Hospital General Universitario Ciudad Real, Ciudad Real, Spain; ${ }^{2}$ Hospital General Universitario Ciudad Real, 13005, Spain

Background: Spondyloarthritis is the term for a group of inflammatory chronic diseases primarily affecting the axial skeleton, as well as the peripheral joints. Regarding bone metabolism in these patients, several studies have reported higher levels of inflammatory activity (BASDAI, BASMI, ESR and CRP) in patients with osteoporosis compared to those without this disease, although no correlations were found.

Objectives: To describe clinical, serological and biological characteristics, as well as bone and mineral metabolism, according to analytical and densitometric criteria in a patient cohort with spondyloarthritis.

Methods: Observational, descriptive and cross-sectional study. A retrospective review was conducted of a database of patients with spondyloarthritis treated during outpatient visits at the Rheumatology Department of Hospital General Universitario de Ciudad Real between June 2018 and June 2019. Variables are described using measures of frequency and of central tendency and dispersion. Results: Cohort of 115 patients (64 men and 51 women). Average age 45.97 years (+/- $13.41 \mathrm{SD})$. Ankylosing spondylitis in 54 patients, psoriatic arthropathy in 24 , spondyloarthropathy associated with inflammatory bowel disease in 8 , undifferentiated spondyloarthritis in 18 and other types of spondyloarthritis in 11. Regarding treatment, $40.88 \%$ of patients received disease-modifying drugs (methotrexate, sulfasalazine, etc.) and $43.4 \%$ received biologic drugs $(86 \%$ anti-TNF alpha, $12 \%$ anti-IL-17 and 2\% anti-IL-12/23). Moreover, 53.04\% had received corticosteroids during some phase of their disease. Vitamin $D$ levels were 23.81 (+/- 10.5 SD) and $77.4 \%$ of patients had a vitamin D deficiency/insufficiency. Of the total cohort, $34.78 \%$ presented osteopenia and $3.58 \%$ osteoporosis (T-Score and Z-Score).

Conclusion: In this study, patients with spondyloarthritis show high percentages of osteopenia and osteoporosis, undiagnosed until this time, along with vitamin $\mathrm{D}$ deficiency. This data suggests higher prevalences of these metabolic bone diseases. Osteoporosis prevention is essential due to the risk of developing early fractures resulting from increased bone fragility.

\section{References:}

[1] Pray C, Feroz NI, Nigil Haroon N. Bone Mineral Density and Fracture Risk in Ankylosing Spondylitis: A Meta-Analysis. Calcif Tissue Int. 2017 Aug;101(2):182-192.

[2] Zhang $\mathrm{M}$ et al. The association between ankylosing spondylitis and the risk of any, hip, or vertebral fracture: A meta-analysis. Medicine (Baltimore). 2017 Dec; 96(50): e8458.

[3] Erten S, Kucuksahin O, Sahin A, Altunoglu A, Akyol M, Koca C. Decreased plasma vitamin $D$ levels in patients with undifferentiated spondyloarthritis and ankylosing spondylitis. Intern Med. 2013;52(3):339-44

[4] Mermerci B, Pekin Dogan Y, Sivas F, Bodur H, Ozoran K. The relation between osteoporosis and vitamin $\mathrm{D}$ levels and disease activity in ankylosing spondylitis. Rheumatol Int 2010;30:375-381

[5] Arends S, Spoorenberg A, Bruyn W, Houtman PM, Leijsma MK, Kallenberg CGM, Brouwer E, van der Veerce. The relation between bone mineral density, bone turnover markers, and vitamin D status in ankylosing spondylitis patients with active disease: a cross-sectional analysis. Osteoporos Int 2011;22:1431-1439.

[6] Lange U, Teichmann J, Strunk J, ller-Ladner U, Schmidt KL. Association of 1.25 vitamin D2 deficiency, disease activity and low bone mass in ankylosing spondylitis.

Disclosure of Interests: None declared

DOI: 10.1136/annrheumdis-2020-eular.1640

\section{AB1298-HPR RHEUMA-VOR: A PROOF-OF-CONCEPT NETWORK STUDY FOR THE IMPROVEMENT OF RHEUMATOLOGICAL HEALTH CARE THROUGH COORDINATED COOPERATION}

M. Dreher ${ }^{1}$, R. E. Schmidt ${ }^{2}$, T. Witte ${ }^{2}$, G. Assmann ${ }^{3}$, K. Hoeper ${ }^{4}$,

K. Triantafyllias ${ }^{5}$, J. Zeidler ${ }^{6}, \mathrm{H}$. Binder ${ }^{7}$, A. Schwarting ${ }^{1,5} .{ }^{1}$ University Medical

Center of the Johannes Gutenberg University Mainz, Division of Rheumatology and Clinical Immunology, Mainz, Germany; ${ }^{2}$ Hannover Medical School,

Department of Immunology and Rheumatology, Hannover, Germany; ${ }^{3}$ Saarland University Medical Center, Rheumatology, Homburg, Germany; ${ }^{4}$ Center for Rheumatology Lower Saxony e.V, Hannover, Germany; ${ }^{5}$ ACURA Center of Rheumatology Rhineland-Palatinate, Bad Kreuznach, Germany; ${ }^{6}$ Leibniz University Hannover, Center for Health Economics Research Hannover, Hannover, Germany; ${ }^{7}$ University Medical Center Freiburg, Institute of Medical Biometry and Statistics, Freiburg, Germany

Background: Rheumatoid arthritis (RA), psoriasis arthritis (PsA) and spondy loarthritis $(\mathrm{SpA})$ are the most common chronic inflammatory rheumatic diseases. For all three diseases, the so-called "window of opportunity" $[1,2,4]$ has been identified as the decisive factor affecting the outcome.

Objectives: The aim of the prospective study is to improve the early diagnosis of RA, PsA and SpA and thus positively impact the quality of care for patients with the support of coordinating centers.

Methods: Primary care providers are given access to screening questionnaires to document potential early cases of RA, PsA, and SpA, based on characteristic symptoms. These are evaluated by multidisciplinary teams at the regional coordinating centers. If they fulfill the criteria for referral, patients get an appointment at a cooperating rheumatology specialist within weeks. If a rheumatic disease is diagnosed, physicians and patients receive questionnaires about sociodemographic, physilogical and psychological parameters.

In order to increase the quality of referrals, a 15-minute rheumatological consultation at the ACURA Rheumatology Center was implemented for all patients from Rhineland-Palatinate whose suspected diagnosis was confirmed by the coordination centre. In course of this, the Rheuma-VOR Screening-App will also be developed and optimized. The cooperating partners and additional information are already published [5]

Results: Preliminary data (31 Dec 2019) are presented. In Rhineland-Palatinate, Saarland and Lower Saxony, 4942 suspected diagnoses of 1526 different referring physicians have now been reported. A total of 2578 patients were referred by the three coordination centres to one of the 49 participating rheumatological specialists. 1004 patients were diagnosed with one of the three diseases. About 306 patients have already taken the follow-up appointment after one year. 